\title{
HYDROGENATED POLYCYCLIC AROMATIC HYDROCARBONS AND THE 2940 AND 2850 WAVENUMBER (3.40 AND 3.51 MICRON) INFRARED EMISSION FEATURES
}

\author{
Max P. Bernstein, Scott A. Sandford, and Louis J. Allamandola \\ Astrophysics Branch, NASA Ames Research Center, Mail Stop 245-6, Moffett Field, CA 94035-1000 \\ Received 1996 August 12; accepted 1996 September 20
}

\begin{abstract}
The $3150-2700 \mathrm{~cm}^{-1}(3.17-3.70 \mu \mathrm{m})$ range of the spectra of a number of Ar-matrix-isolated PAHs containing excess $\mathrm{H}$ atoms $\left(\mathrm{H}_{n}-\mathrm{PAHs}\right)$ are presented. This region covers features produced by aromatic and aliphatic $\mathrm{C}-\mathrm{H}$ stretching vibrations as well as overtone and combination bands involving lower lying fundamentals. The aliphatic $\mathrm{C}-\mathrm{H}$ stretches in molecules of this type having low to modest excess $\mathrm{H}$ coverage provide excellent fits to a number of the weak emission features superposed on the plateau between 3080 and $2700 \mathrm{~cm}^{-1}(3.25$ and $3.7 \mu \mathrm{m})$ in the spectra of many planetary nebulae, reflection nebulae, and $\mathrm{H}$ in regions. Higher $\mathrm{H}$ coverage is implied for a few objects. We compare these results in context with the other suggested identifications of the emission features in the $2950-2700 \mathrm{~cm}^{-1}(3.39-3.70 \mu \mathrm{m})$ region and briefly discuss their astrophysical implications.
\end{abstract}

Subject headings: $\mathrm{H}$ II regions - infrared: ISM: lines and bands - ISM: molecules - molecular processes planetary nebulae: general

\section{INTRODUCTION}

The $3125-2780 \mathrm{~cm}^{-1}(3.20-3.60 \mu \mathrm{m})$ spectrum of objects which emit the well-known infrared features at 3040, 1610, 1300,1160 , and $885 \mathrm{~cm}^{-1}(3.29,6.2,7.7,8.6$, and $11.3 \mu \mathrm{m})$ has three components: a band at $3040 \mathrm{~cm}^{-1}(3.29 \mu \mathrm{m}$, generally most intense), a broad, weak pedestal from about 3125$2700 \mathrm{~cm}^{-1}$, and a series of features on the pedestal that are usually weaker than the $3040 \mathrm{~cm}^{-1}$ band (Geballe et al. 1985; de Muizon et al. 1986; Nagata et al. 1988; Geballe et al. 1989; Jourdain de Muizon, d'Hendecourt, \& Geballe 1990a; Roche et al. 1996). Within the framework of the polycyclic aromatic hydrocarbon (PAH) model, these bands all arise from free, vibrationally excited PAHs (Allamandola, Tielens, \& Barker 1989; Puget \& Leger 1989). While it is thought that the $3040 \mathrm{~cm}^{-1}$ component is due to the aromatic $\mathrm{C}-\mathrm{H}$ stretch of PAHs, the precise identification of the weak features just longward of the $3040 \mathrm{~cm}^{-1}$ emission feature has remained somewhat enigmatic. These features fall near $2940,2890,2850$, and $2810 \mathrm{~cm}^{-1}(3.40,3.46,3.51$, and $3.56 \mu \mathrm{m})$ and are superposed on the "plateau." In most emission objects that produce the emission band family, these features are weak compared to the $3040 \mathrm{~cm}^{-1}(3.29 \mu \mathrm{m})$ band and show a tendency to decrease in strength with decreasing frequency (cf. Geballe et al. 1985; de Muizon et al. 1986; Nagata et al. 1988; Geballe et al. 1989; Jourdain de Muizon et al. 1990a; Roche et al. 1996). However, observations of a small number of earlytype objects spanning the evolutionary bridge between carbonrich giants and planetary nebulae show that the 2940 and $2850 \mathrm{~cm}^{-1}$ bands $(3.40$ and $3.5 \mu \mathrm{m}$ ) can actually be stronger than the $3040 \mathrm{~cm}^{-1}$ feature (Geballe \& van der Veen 1990; Geballe et al. 1992).

Several identifications for these features have been suggested. These include overtones and combinations of lower frequency PAH vibrational modes and "hot" bands associated with relaxation from higher vibrational levels which are anharmonic (Barker, Allamandola, \& Tielens 1987; Allamandola et al. 1989), the $\mathrm{C}-\mathrm{H}$ stretching vibrations of aliphatic side groups on PAHs (Jourdain de Muizon, d'Hendecourt, \&
Geballe 1990b), and PAHs containing excess $\mathrm{H}$ atoms ( H $_{n}$-PAHs) (Schutte, Tielens, \& Allamandola 1993). All the suggested possibilities probably contribute to the emission, the extent of each depending on conditions in the local environment. Here we provide laboratory data and comparisons which show that $\mathrm{H}_{n}$-PAHs are very attractive candidates for these features. $\mathbf{H}_{n}$-PAHs are PAHs that have a few additional hydrogen atoms attached, principally to the peripheral carbon atoms. These are particularly attractive candidates, since many of the emission regions are rich in $\mathrm{H}$ atoms.

The methods used in this study have been described in detail elsewhere (Hudgins, Sandford, \& Allamandola 1994; Hudgins \& Allamandola 1995). Samples were isolated in argon matrices (Ar/sample typically on the order of 1000$)$ on a CsI window at $12 \mathrm{~K}$, and their infrared spectra were measured over the $5000-500 \mathrm{~cm}^{-1}(2-20 \mu \mathrm{m})$ range at a resolution of $0.9 \mathrm{~cm}^{-1}$.

In the following sections we compare the $3150-2700 \mathrm{~cm}^{-1}$ $(3.17-3.70 \mu \mathrm{m})$ spectra of a number of $\mathbf{H}_{n}-\mathrm{PAHs}$ with the interstellar infrared emission, demonstrate that molecules of this type are attractive candidates for some of the emission in this spectral region, and discuss a few of the implications of this work.

\section{EXPERIMENTAL RESULTS}

We designate PAHs with excess $\mathbf{H}$ as $\mathbf{H}_{n}-\mathrm{PAHs}$, where $n$ is the number of $\mathrm{H}$ atoms in excess of those needed to make the fully aromatic structure. These extra hydrogen atoms convert specific $s p^{2}$ bonded, planar, aromatic $\mathrm{C}-\mathrm{H}$ groups (with stretching vibrations near $\left.3050 \mathrm{~cm}^{-1}\right)$ to $s p^{3}$ bonded, tetragonal, aliphatic $\mathrm{C}-\mathrm{H}$ groups (with stretching vibrations in the $2990-2780 \mathrm{~cm}^{-1}$ region; Bellamy 1960). The addition of extra $\mathrm{H}$ atoms to a PAH forces the localization of the $\pi$ electrons, removes aromaticity from the rings affected, and introduces ring strain. For excess hydrogen atoms attached to a peripheral carbon (a carbon bonded to two other carbon atoms), secondary $\left(-\mathrm{CH}_{2}-\right) \mathrm{C}-\mathrm{H}$ stretches are expected. Extra hydrogens bonded to internal carbon atoms (which are bonded to three 


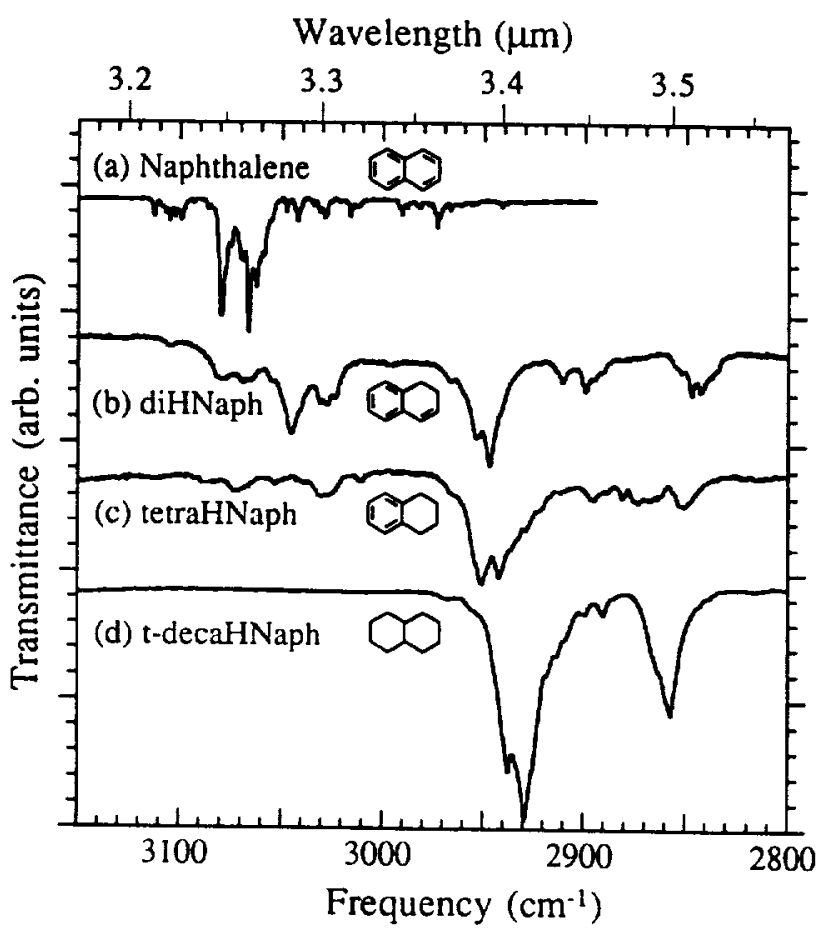

Fig. 1.-The $3150-2800 \mathrm{~cm}^{-1}(3.17-3.57 \mu \mathrm{m})$ laboratory infrared spectra of the PAH naphthalene and associated $\mathrm{H}_{n}$-PAHs having increasingly higher excess $\mathrm{H}$ coverage. In order, the spectra are of $(a)$ naphthalene, $(b)$ dihydronaphthalene (diHNaph), (c) tetrahydronaphthalene (tetraHNaph), and (d) trans-decahydronaphthalene (t-decaHNaph). All spectra are of molecules isolated in $\mathrm{Ar}$ matrices at $12 \mathrm{~K}$ with $\mathrm{Ar} / \mathrm{H}_{n}-\mathrm{PAH} \approx 1000$.

other carbon atoms) should produce a tertiary $(-\mathrm{C}-\mathrm{H})$, or diamond-like, $\mathrm{C}-\mathrm{H}$ stretch.

$\mathrm{H}_{n}$-PAHs are the intermediates between fully aromatic and fully aliphatic cyclic structures. The aromatic and aliphatic classes possess distinct infrared characteristics, and the infrared spectra of $\mathrm{H}_{n}-\mathrm{PAHs}$ reflect their mixed nature by showing both aromatic and aliphatic signatures, the extent of each depending on the degree of excess hydrogenation. A detailed discussion of the entire mid-infrared spectrum $\left(4000-400 \mathrm{~cm}^{-1}\right.$, 2.5-25 $\mu \mathrm{m}$ ) of these molecules will be presented elsewhere (Bernstein, Sandford, \& Allamandola 1997). We focus here on the $\mathrm{C}-\mathrm{H}$ stretch region $3150-2700 \mathrm{~cm}{ }^{i}(3.17-3.70 \mu \mathrm{m})$. Figure 1 shows the spectral evolution in this region for the $P A H$ naphthalene $\left(\mathrm{C}_{8} \mathrm{H}_{10}\right)$ as it undergoes hydrogen addition.

A number of points are immediately apparent from these spectra. First, the top trace is dominated by the well-known aromatic band near $3050 \mathrm{~cm}^{-1}$, and the addition of extra $\mathrm{H}$ to PAHs produces a number of new features in the $2990-$ $2780 \mathrm{~cm}^{-1}(3.35-3.60 \mu \mathrm{m})$ region characteristic of the $\mathrm{C}-\mathrm{H}$ stretch in aliphatics. The exact number and position of these features varies from one molecule to another with $\mathrm{H}$ atom coverage and location, but the two strongest new features in all the $\mathbf{H}_{n}$-PAHs we have studied to date fall in the vicinity of 2940 and $2850 \mathrm{~cm}^{-1}(3.40$ and $3.51 \mu \mathrm{m})$. These bands are due to the asymmetric and symmetric $\mathrm{C}-\mathrm{H}$ stretching vibrations, respectively, of the "aliphatic" $-\mathrm{CH}_{2}-$ groups in the $\mathrm{H}_{n}$-PAHs. Second, with the addition of more $\mathrm{H}$, the aliphatic features grow in strength while the aromatic $\mathrm{C}-\mathrm{H}$ stretching features decrease. The 2940 and $2850 \mathrm{~cm}^{-1}$ features are typically as strong as the aromatic $\mathrm{C}-\mathrm{H}$ stretching feature after the addition of only a few $\mathrm{H}$ atoms, and they quickly dominate the $\mathrm{C}-\mathrm{H}$ stretching region upon further $\mathrm{H}$ atom addition. This is because the intrinsic band strength of the aliphatic $\mathrm{C}-\mathrm{H}$

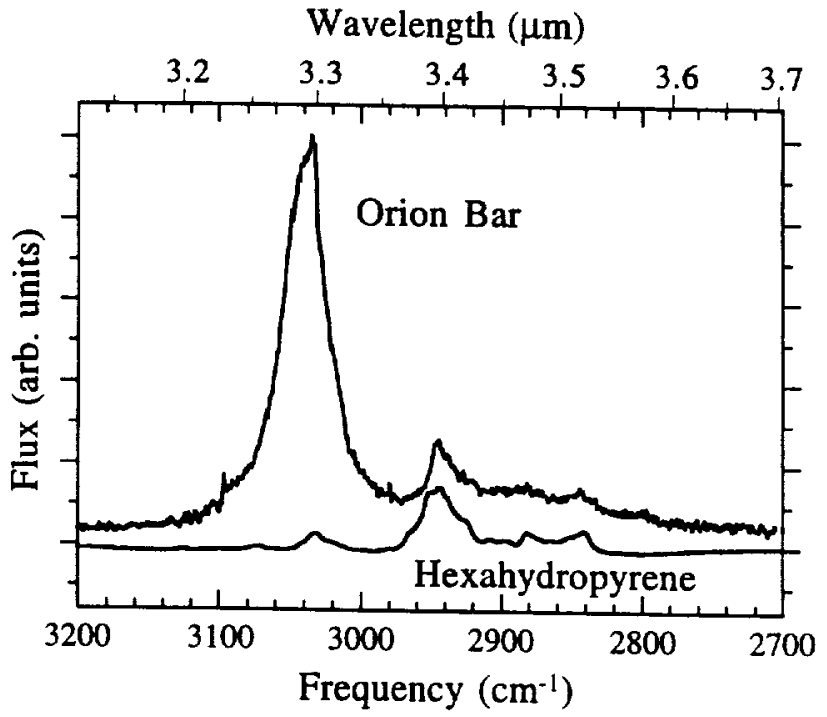

Fic. 2.-Comparison of the $3200-2700 \mathrm{~cm}^{-1}(3.13-3.70 \mu \mathrm{m})$ emission spectrum of position 4 in the Orion bar (from Sloan et al. 1997) with the absorption spectrum of matrix-isolated hexahydropyrene. This $\mathrm{H}_{n}-\mathrm{PAH}$ provides a reasonable match to the 2940,2890 , and $2850 \mathrm{~cm}^{-1}(3.40,3.46$, and $3.51 \mu \mathrm{m}$ ) emission features. The dominance of the aliphatic over the aromatic features in $\mathrm{H}_{n}-\mathrm{PAH}$ spectra shows that, as a class, they contribute very little to the aromatic $\mathrm{C}-\mathrm{H}$ stretch near $3040 \mathrm{~cm}^{-1}(3.29 \mu \mathrm{m})$.

stretch near $2940 \mathrm{~cm}^{-1}$ is typically $2-3$ times stronger than the aromatic feature near $3050 \mathrm{~cm}^{-1}$ (Wexler 1967), and an external aromatic carbon bears one $\mathrm{H}$ atom whereas an aliphatic carbon carries two. Thus, $\mathrm{H}_{n}$-PAHs having only modest $\mathrm{H}$ excesses will have aromatic and aliphatic stretching features of comparable strength even though most of their carbon atoms are in aromatic structures.

While the $\mathrm{C}-\mathrm{H}$ stretching bands produced by $\mathrm{H}_{n}$-PAHs show both their aromatic and aliphatic natures, the aliphatic bands do not fall exactly at the $-\mathrm{CH}_{2}-$ frequencies characteristic of completely aliphatic species. They are slightly shifted either up or down in frequency from their normal positions. This is probably due to inductive effects and the presence of molecular strain caused by the proximity of the aromatic moieties within the $\mathrm{H}_{n}$-PAHs. The direction and amount of the shifts vary with $\mathrm{H}$ atom coverage and location on different $\mathrm{H}_{n}$-PAHs.

\section{SPECTRAL COMPARISONS BETWEEN $\mathrm{H}_{n}$-PAHs AND THE INTERSTELL.AR INFRARED EMISSION FEATURES}

Comparison of $\mathrm{H}_{n}$-PAH spectra to the interstellar infrared emission spectra associated with objects representative of both high- and low-excitation environments shows that $\mathbf{H}_{n}$-PAHs likely contribute significantly to the features in the 3000$2800 \mathrm{~cm}^{-1}(3.33-3.57 \mu \mathrm{m})$ region and place constraints on the possible interstellar $\mathrm{H}_{n}$-PAH populations.

\section{1. $\mathrm{H}_{n}-P A H s$ and the lonization Ridge in Orion}

Figure 2 shows a comparison of the $3200-2700 \mathrm{~cm}$ ' $(3.13-3.70 \mu \mathrm{m})$ emission spectrum of the ionized region of the Orion bar (Sloan et al. 1997) with the absorption spectrum of matrix-isolated hexahydropyrene $\left(H_{6}\right.$-pyrene $)$. The Orion bar lies at the interface of an $\mathrm{H}$ il region with a dense molecular cloud (Tielens et al. 1993; Sloan et al. 1996). The 3200$2700 \mathrm{~cm}^{-1}$ spectrum of the Orion bar (Geballe et al. 1989) is very similar to that of other emission objects associated with high-energy, high-flux UV excitation fields, including compact 
objects, such as IRAS $21282+5050$ (de Muizon et al. 1986) and planetary nebulae (Roche et al. 1996), which are not associated with molecular clouds. These objects exhibit the most common emission profile in this spectral region, i.e., a relatively strong aromatic $\mathrm{C}-\mathrm{H}$ stretching band near $3040 \mathrm{~cm}^{-1}(3.29 \mu \mathrm{m})$ followed by considerably weaker emission features near $2940,2890,2850$, and $2810 \mathrm{~cm}^{-1}(3.40,3.46$, 3.51 , and $3.56 \mu \mathrm{m}$ ) superposed on a broad plateau.

Figure 2 shows that hexahydropyrene provides a good match to the 2940,2890 , and $2850 \mathrm{~cm}^{-1}(3.40,3.46$, and $3.51 \mu \mathrm{m})$ features. When one takes the approximately $10 \mathrm{~cm}{ }^{1}$ redshift expected for emission from highly vibrationally excited species (Joblin et al. 1995 and references therein) into account, the match is even further improved. Figure 2 also demonstrates that, because of the dominance of the aliphatic features intrinsic to $\mathrm{H}_{n}$-PAH spectra, they will contribute very little to the aromatic $\mathrm{C}-\mathrm{H}$ stretching feature near $3040 \mathrm{~cm}{ }^{1}(3.29 \mu \mathrm{m})$ even if they provide most of the emission in the 2950$2700 \mathrm{~cm}^{1}(3.39-3.70 \mu \mathrm{m})$ region. Since the aliphatic $\mathrm{C}-\mathrm{H}$ stretching bands are among the strongest features produced by $\mathrm{H}_{n}$-PAHs and these features are generally weak in highexcitation objects, these molecules should contribute only minor flux to the interstellar emission below $2000 \mathrm{~cm}^{-1}$ $(\lambda>5 \mu \mathrm{m})$ and most of the total emission from these objects is still produced by "normal" PAHs (Bernstein et al. 1997).

Similar comparisons between the spectra of other $\mathrm{H}_{n}$-PAHs and that of the Orion bar indicate that almost all $\mathrm{H}_{n}$-PAHs with modest excess $\mathrm{H}$ coverage do a similarly good job of fitting the interstellar 2940 and $2850 \mathrm{~cm}^{-1}(3.40$ and $3.51 \mu \mathrm{m})$ features, although not all of them produce the $2890 \mathrm{~cm}$ $(3.46 \mu \mathrm{m})$ feature. However, $\mathrm{H}_{n}$-PAHs with large $\mathrm{H}$ excesses do not fit as well. Figure 3 illustrates these points. Spatial studies by Sloan et al. (1997) show that the $2940 \mathrm{~cm}^{-1}$ (3.41 $\mu \mathrm{m})$ feature that best matches the $\mathrm{H}_{n}$-PAH spectra peaks in the zone in the Orion bar where the $\mathrm{H}$ atom abundance reaches a maximum. This is traced out by the $\mathrm{H}_{2}$ emission which lies precisely at the interface between the ionized $(\mathrm{H} \mathrm{II})$ and neutral $(\mathrm{H} \mathrm{I})$ regions. This behavior is consistent with $\mathrm{H}_{n}$-PAHs being produced by the addition of $\mathrm{H}$ atoms in this zone and surviving up to the photodissociation region, as well as (perhaps) with $\mathrm{H}_{n}$-PAHs being liberated from interstellar ices at the cloud $H$ II region interface.

Another possible contributor to these weaker emission features are the $\mathrm{C}-\mathrm{H}$ stretching vibrations in aliphatic side groups, such as methyl $\left(-\mathrm{CH}_{3}\right)$ and ethyl $\left(-\mathrm{CH}_{2} \mathrm{CH}_{3}\right)$ groups, on PAHs (Jourdain de Muizon et al. 1990b). In this case, $-\mathrm{CH}_{3}$ seemed most promising for the $2940 \mathrm{~cm}^{-1}(3.4 \mu \mathrm{m})$ feature (Jourdain de Muizon et al. 1990b; Sandford 1991). To date, the best laboratory fit to the interstellar $2940 \mathrm{~cm}^{-1}$ (3.4 $\mu \mathrm{m})$ feature has been provided by the spectrum of gas-phase methyl coronene (Joblin 1992). Figure 3 also presents the spectrum of methyl coronene from Joblin (1992) for comparison. While the aliphatic $\mathrm{C}-\mathrm{H}$ stretch in methyl coronene produces a good match to the $2940 \mathrm{~cm}^{-1}(3.4 \mu \mathrm{m})$ feature, the fits provided by the $\mathrm{H}_{n}$-PAHs to these interstellar spectra are better, particularly when one takes the approximately $10 \mathrm{~cm}^{-1}$ redshift expected for emission from vibrationally cxcited PAHs into account (Joblin et al. 1995).

\section{2. $\mathrm{H}_{n}-P A H s$ and the Protoplanetary Nebula IRAS $05341+0852$}

Figure 3 also compares the $3000-2800 \mathrm{~cm}^{-1}(3.3-3.6 \mu \mathrm{m})$ absorption spectra of $\mathrm{H}_{n}$-PAHs and methyl coronene with the emission spectrum of IRAS $05341+0852$ (hereafter IRAS 05341 ), an optically visible star that has left the asymptotic

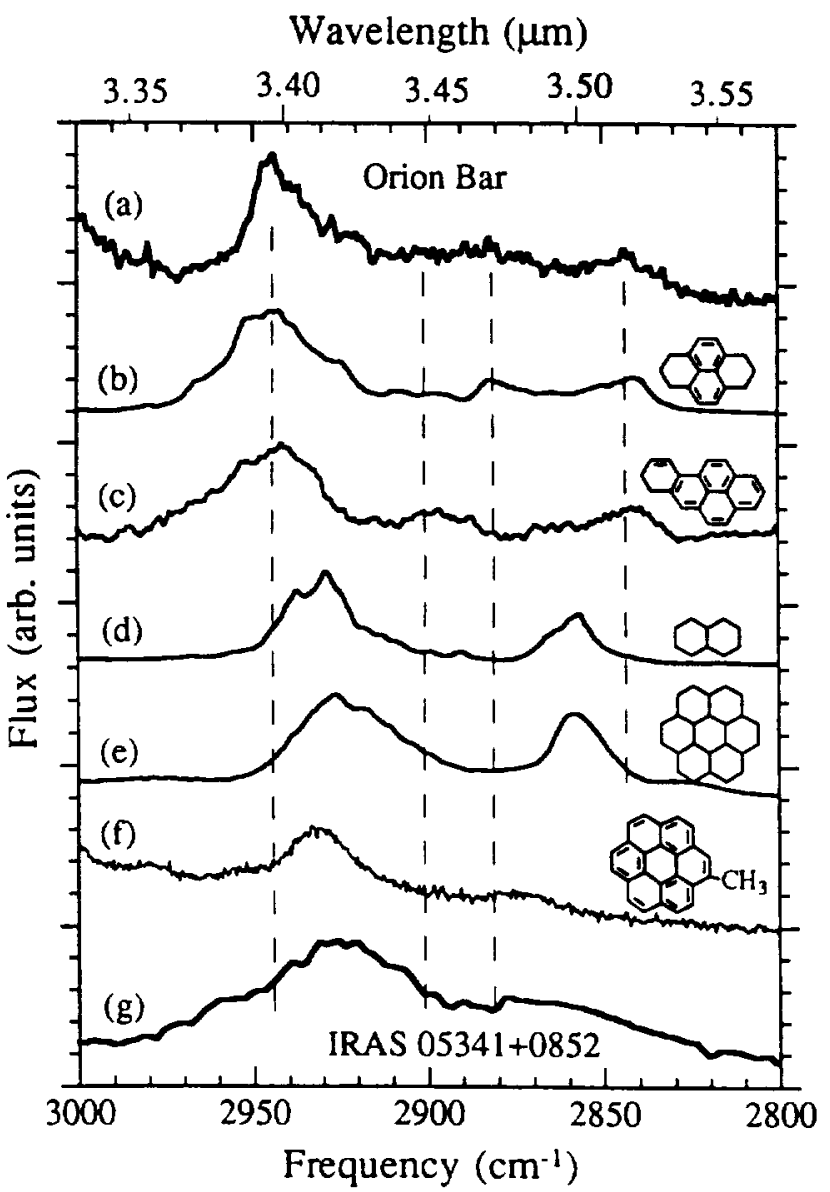

Fici. 3.-Comparison of the $3000-2800 \mathrm{~cm}^{-1}(3.33-3.57 \mu \mathrm{m})$ emission spectra of the Orion bar and JRAS 05341 with the spectra of several $\mathrm{H}_{n}$-PAHs and methyl coronene. In order, the spectra are of $(a)$ the Orion bar (Sloan et al. 1997) (b) hexahydropyrene, (c) 7,8 dihydrobenzolalpyrene, (d) transdecahydronaphthalene, $(e)$ perhydrocoronene, $(f)$ methyl coronene (taken from Joblin 1992), and (g) IRAS 05341 (Geballe \& van der Veen 1990). In general, $\mathrm{H}_{n}$-PAHs with only modest excess $\mathrm{H}$ coverage provide a good match to the emission from high-excitation zones such as the transition region in Orion, while methyl-PAHs and $\mathrm{H}_{n}$-PAHs with high excess $\mathrm{H}$ coverage provide a better match to more benign environments like that in IRAS 05341 .

giant branch and is thought to be a protoplanetary nebula (Geballe \& van der Veen 1990). While the aromatic $3040 \mathrm{~cm}^{\prime \prime}$ $(3.29 \mu \mathrm{m})$ emission feature dominates in Orion (Fig. 2), the aliphatic features peaking near 2925 and $2870 \mathrm{~cm}^{-1}(3.42$ and $3.48 \mu \mathrm{m})$ are actually stronger than the aromatic band in the spectrum of IRAS 05341. In addition, the two strongest features fall at positions slightly different than those of the more common emission objects.

Given that the emission bands from IRAS 05341 fall at slightly different positions than those from most other objects, it is not surprising that the $\mathbf{H}_{n}$-PAHs that provide an excellent fit to the features in the spectrum of the Orion bar do not fit as well to the spectrum of IRAS 05341 , although they do fall within the overall envelope of the emission features and could contribute to the "shoulder" near $2960 \mathrm{~cm}^{-1}(3.38 \mu \mathrm{m})$. Nonetheless, none of the $\mathrm{H}_{n}-\mathrm{PAHs}$ with modest $\mathrm{H}$ atom excesses in our database produce features that fall directly at the $2925 \mathrm{~cm}^{-1}(3.42 \mu \mathrm{m})$ position of the dominant peak in the spectrum of IRAS 05341 . Instead, the $\mathrm{H}_{n}$-PAHs that provide the best fits to the spectrum of IRAS 05341 are the cyclic aliphatics, namely, those with the highest $\mathrm{H}$ atom cxcesses.

The implied difference in the relative $H_{n}-\mathrm{PAH} / \mathrm{PAH}$ pop- 
ulations of objects such as IRAS 05341 and objects like the Orion bar is understandable in terms of the differences in the environments and ages of these two objects. IRAS 05341 falls at a much earlier stage of evolution than a fully evolved planetary nebula such as NGC 7027, and it has a more benign UV radiation environment than mature planetary nebulae or the interfaces of $\mathrm{H}$ II regions. Thus, less stable carbonaceous species like $\mathrm{H}_{n}-\mathrm{PAHs}$ and methyl-PAHs can better survive there. "Normal" PAHs should also survive in such regions. Indeed, since $\mathrm{H}_{n}$-PAHs with large excess $\mathrm{H}$ coverage produce very little absorption at the aromatic $\mathrm{C}-\mathrm{H}$ stretching position (or none, in the case of the perhydrogenated, or fully aliphatic species), the presence of any aromatic $3040 \mathrm{~cm}^{-1}$ emission feature in IRAS 05341 implies that substantial numbers of normal PAHs also lie along this line of sight.

Note that the feature produced by gas-phase methyl coronene (Joblin 1992) provides an excellent fit to the $2925 \mathrm{~cm}^{-1}$ $(3.42 \mu \mathrm{m})$ position of the feature in IRAS 05341, one that is clearly better than that to the features in high-excitation objects such as NGC 7027 and the Orion bar. It matches the central position of the IRAS 05341 feature better than most of the $\mathrm{H}_{n}$-PAHs having modest excess $\mathrm{H}$ coverage, and is similar to the match provided by the completely cyclic aliphatic species, with some differences in the vicinity of the emission between 2880 and $2840 \mathrm{~cm}^{-1}$. As with the $\mathrm{H}_{n}$-PAHs having large excess $\mathrm{H}$ coverage, the better fit of methyl coronene to IRAS 05341 is consistent with its more benign environment.

\section{CONCLUSION}

The addition of $\mathrm{H}$ atoms to PAHs converts aromatic rings in the molecules into aliphatic rings. This results in a decrease in the strength of the aromatic $\mathrm{C}-\mathrm{H}$ stretching band and the creation of new aliphatic $\mathrm{C}-\mathrm{H}$ stretching bands, the strongest of which fall near 2940 and $2850 \mathrm{~cm}^{-1}(3.40$ and $3.51 \mu \mathrm{m})$. Because of their larger intrinsic strengths and larger $\mathrm{H}$-to-C ratio, the aliphatic $\mathrm{C}-\mathrm{H}$ stretches quickly dominate the $\mathrm{C}-\mathrm{H}$ stretching spectral region even for molecules that are primarily aromatic. The intimate connection of the aromatic to aliphatic domains in $\mathrm{H}_{n}$-PAHs often results in molecular strain and inductive effects that displace both the aromatic and aliphatic $\mathrm{C}-\mathrm{H}$ stretches from their normal characteristic frequencies. The more excess $\mathrm{H}$ added to a PAH, the less aromatic and more aliphatic it becomes.

The good fits to the interstellar emission data provided by the $\mathrm{H}_{n}$-PAHs in Figures 2 and 3 suggest that molecules in this class are reasonable contributors to the interstellar emission between 2990 and $2780 \mathrm{~cm}^{-1}(3.35-3.60 \mu \mathrm{m}) . \mathrm{H}_{n}$-PAHs are not as stable as their parent PAHs and are readily prone to losing their excess $\mathrm{H}$ upon absorption of high-energy photons in order to regain the thermodynamic stabilization energy provided by fully aromatic structures. Thus, variations in the aromatic and aliphatic features near 3040 and $2940 \mathrm{~cm}^{-1}$ (3.29 and $3.40 \mu \mathrm{m}$ ), respectively, are expected and should follow environmental conditions such as the availability of $\mathbf{H}$ atoms and the intensity of the local UV radiation. The implied increase in importance of $\mathrm{H}_{n}$-PAHs, and their higher excess $\mathrm{H}$ coverage, in early-type objects such as IRAS 05341 over the Orion bar and NGC 7027 is consistent with this picture.

Since the emission features attributed to $\mathrm{H}_{n}-\mathrm{PAHs}$ are among the weakest in the interstellar spectrum, yet the strongest in the laboratory data, the implied amounts of $\mathrm{H}_{n}$-PAHs are small relative to fully aromatic species (PAHs). Most of the emission in the $\mathrm{C}-\mathrm{H}$ stretch region from most of the objects is still produced by neutral and/or ionized PAHs, not $\mathrm{H}_{n}$-PAHs. While objects like IRAS 05341 contain higher relative abundances of $\mathbf{H}_{n}$-PAHs or methyl-PAHs, the presence of abundant "normal" PAHs is still required to account for the rest of the spectrum. Thus, the identification of the $2940 \mathrm{~cm}^{-1}$ emission feature with $\mathrm{H}_{n}-\mathrm{PAHs}$ is not expected to significantly affect previous estimates of the $\mathrm{C}$ abundances in these objects.

The good fits provided to the interstellar emission spectra by $\mathrm{H}_{n}$-PAHs and their promise as unique probes of specific environments argue that further tests of their existence in space should be vigorously pursued. Additional spatial-spectral studies similar to those of Geballe et al. (1989), Joblin et al. (1996), and Sloan et al. (1997) are warranted. Searches at longer wavelengths for bands characteristic of $\mathrm{H}_{n}$-PAHs are also warranted. For example, since aliphatic deformation modes fall near $1470 \mathrm{~cm}^{-1}(6.8 \mu \mathrm{m})$, searching for correlations and band-strength variations between the interstellar 2940 and $1470 \mathrm{~cm}^{-1}$ emission bands could provide useful insights. The laboratory spectra of a number of $\mathrm{H}_{n}$-PAHs are also needed in order to better understand the spectral characteristics of these molecules in both their neutral and ionized forms. We are currently carrying out such a study and will report on this work in the near future (Bernstein et al. 1997).

We wish to thank Christine Joblin and Tom Geballe for kindly sending us the electronic versions of their spectra. The authors are also grateful for useful discussions with D. Hudgins and G. Sloan and, as always, for excellent technical support from $R$. Walker. This work was supported by NASA grants 188-44-57-01 (Astrophysics), 452-22-94-06 (Origins of Solar Systems Program), and 185-52-12-04 (Exobiology Program).

\section{REFERENCES}

Allamandola, L. J., Tielens, A. G. G. M., \& Barker, J. R. 1989, ApJS, 71, 733 Barker, J. R., Allamandola, L. J., \& Tielens, A. G. G. M. 1987, ApJ, 315, L61 Bellamy, L. J. 1960, The Infrared Spectra of Complex Molecules (New York: Wiley)

Bernstein, M. P., Sandford, S. A., \& Allamandola, L. J. 1997, in preparation de Muizon, M., Geballe, T. R., d'Hendecourt, L. B., \& Baas, F. 1986, ApJ, 306, L105

Geballe, T. R., Lacy, J. H., Persson, S. E., McGregor, P. J., \& Soifer, B. T. 1985, ApJ, 292, 500

Geballe, T. R., Tielens, A. G. G. M., Allamandola, L. J., Moorhouse, A., \& Brand, P. W. J. L. 1989, ApJ, 341, 278

Geballe, T. R., Tielens, A. G. G. M., Kwok, S., \& Hrivnak, B. J. 1992, ApJ, 387, L89

Geballe, T. R., \& van der Veen, W. E. C. J. 1990, A\&A, 235, L9

Hudgins, D. M., \& Allamandola, L. J. 1995, J. Phys. Chem., 99, 3033

Hudgins, D. M., Sandford, S. A., \& Allamandola, L. J. 1994, J. Phys. Chem., 98, 4243

Joblin, C. 1992, Ph.D. thesis, Univ. Paris

Joblin, C., Boissel, P., Leger, A., d'Hendecourt, L. B., \& Defourneau, D. 1995, A\&A, 299, 835
Joblin, C., Tielens, A. G. G. M., Allamandola, L. J., \& Geballe, T. R. 1996, ApJ, 458,610

Jourdain de Muizon, M., d'Hendecourt, L. B., \& Geballe, T. R. 1990a, A\&A 227,526

227, $1990 \mathrm{~b}, \mathrm{~A} \& \mathrm{~A}, 235,367$

Nagata, T., Tokunaga, A. T., Sellgren, K., Smith, R. G., Onaka, T., Nakada, Y., \& Sakata, A. 1988, ApJ, 326, 157

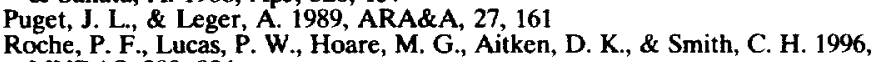
MNRAS, 280,924

Sandford, S. A. 1991, ApJ, 376, 599

Schutte, W. A., Tielens, A. G. G. M., \& Allamandola, L. J. 1993, ApJ, 415, 397

Sloan, G. C., Bregman, J. D., Geballe, T. R., Allamandola, L. J., \& Woodward, C. E. 1997, ApJ, 474, in press

Tielens, A., Meixner, M. M., van der Werf, P. P., Bregman, J., Tauber, J. A., Stutzki, J., \& Rank, D. 1993, Science, 262, 86

Wexler, A. S. 1967, Appl. Spectrosc. Rev., 1, 29 